\title{
Effects of the endothelial nitric oxide synthase gene polymorphisms on the risk of metabolic syndrome in subjects with schizophrenia
}

\author{
D. Parshukova*, N. Fattakhov, L. Smirnova, V. Dubrovskaya, E. Kornetova, \\ A. Semke, S. Ivanova \\ Mental Health Research Institute, Tomsk, Russia \\ *e-mail: Susl2008@yandex.ru
}

\begin{abstract}
Key words: schizophrenia, metabolic syndrome, endothelial nitric oxide synthase, single nucleotide polymorphism
\end{abstract}

Motivation and Aim: The frequency of metabolic syndrome (MetS) is significantly higher in schizophrenia ( $\mathrm{SCH}$ ) patients, when compared to the general population [1]. Genetic variation within the endothelial nitric oxide synthase gene (NOS3) may result in impaired endogenous nitric oxide formation and has been associated with cardiovascular diseases [2]. There is growing evidence that polymorphisms in NOS3 influence the development of MetS, however, there is also a controversy regarding the association of polymorphisms in the gene encoding NOS3 and MetS in patients with schizophrenia [3]. The aim of this study was to evaluate whether genetic variants in NOS3 gene could be associated with the risk of MetS in $\mathrm{SCH}$ patients from Russian population.

Methods and Algorithms: 70 Caucasian patients with schizophrenia and MetS and 127 schizophrenic patients with normal BMI were enrolled in the study and genotyped for T-786C (rs2070744), G894T (rs1799983) and C774T (rs1549758) in NOS3. MetS was diagnosed using International Diabetes Federation (IDF) criteria.

Results: The allelic and genotypic frequencies of rs2070744 (promoter region) polymorphism in schizophrenic patients with MetS were significantly different from those in schizophrenic patients with normal BMI. These patients had significantly higher frequencies of rs $2070744 \mathrm{~T}$ allele $\left(\chi^{2}=6.80 ; p=0.009, \mathrm{OR}=0.59 ; 95 \% \mathrm{CI}: 0.40-0.88\right)$, rs2070744 C allele $\left(\chi^{2}=6.80 ; p=0.009, \mathrm{OR}=1.69 ; 95 \%\right.$ CI: $\left.1.14-2.51\right)$ and $\mathrm{rs} 2070744$ TT genotype ( $p=0.006, \mathrm{OR}=0.45 ; 95 \% \mathrm{CI}$ : $0.25-0.82$ ). Strong linkage disequilibrium between rs1799983 and rs1549758 was observed (D’>0.9). No association was observed between NOS3 haplotypes and MetS risk in patients with schizophrenia.

Conclusion: Based on these results, we can conclude that the promoter T-786C variant of NOS3 gene may be considered as a potential genetic marker of increased risk of MS in patients with schizophrenia and may serve as a prognostic biomarker for MetS among Russian schizophrenic subjects.

Acknowledgements: Supported by the program of the Presidium of the Russian Academy of Sciences No. 1.42 "Fundamental research for biomedical technologies".

\section{References}

1. Malan-Müller S. et al. (2015) A systematic review of genetic variants associated with metabolic syndrome in patients with schizophrenia. Schizophr Res. 170(1):1-17.

2. Huang P.L. (2009) eNOS, metabolic syndrome and cardiovascular disease. Trends Endocrinol Metab. 20(6):295-302.

3. Burghardt K. et al. (2014) Endothelial nitric oxide synthetase genetic variants, metabolic syndrome and endothelial function in schizophrenia, J Psychopharmacol, 28(4):349-356. 